\title{
CAD Interfaces to the ARROW Manufactured Product Server
}

\author{
Robert Amor and Leonard Newnham \\ Building Research Establishment, Bucknalls Lane, Garston, Watford WD2 7JR, UK
}

Key words: computer-aided design, product retrieval

\begin{abstract}
The UK national project ARROW (Advanced Reusable Reliable Objects Warehouse) (Newnham et al. 1997, 1998a and 1998b) provides an Internet based framework through which it is possible to identify any of a range of manufactured products meeting specific design criteria. This open framework (based upon the IAI's IFCs) provides a mechanism for users to search for products from any participating manufacturer or supplier based both on specific attributes of a product or on any of the textual descriptions of the product. The service returns the closest matching products and allows the user to navigate to related information including manufacturer, suppliers, CAD details, VR displays, installation instructions, certificates, health and safety information, promotional information, costings, etc. ARROW also provides a toolkit to enable manufacturers and suppliers to more easily map and publish their information in the format utilised by the ARROW system.
\end{abstract}

As part of the ARROW project we have examined the ability to interface from a design tool through to ARROW to automatically retrieve information required by the tool. This paper describes the API developed to allow CAD and simulation tools to communicate directly with ARROW and identify appropriate manufactured information. The demonstration system enables CAD systems to identify the closest matching manufactured product to a designed product and replacing the designed product with the details supplied by the manufacturer for the manufactured product as well as pulling through product attributes utilised by the design application.

This paper provides a description of the ARROW framework and issues faced in providing information based upon standards as well as containing information not currently modelled in public standards. The paper looks at issues of enabling manufacturers and suppliers to move from their current 
world-view of product information to a more data-rich and user accessible information repository (even though this enables a uniform comparison across a range of manufacturer's products). Finally the paper comments on the likely way forward for ARROW like systems in providing quality information to end users.

\section{THE ARROW PROJECT}

ARROW is a UK initiative that can provide access to any construction manufacturer's product information through virtual warehouses. This enables designers and specifiers to correctly identify products that match the parameters of their particular design. Benefits of this approach will be reduced abortive design work and reworking, a more reliable design specification, designs that can be constructed quicker, greater use of off-the-shelf components, savings in cost, and improved response time for customers. To make ARROW feasible a central search engine able to handle structured data and also free-text information has been developed. This is based on data collected from all manufacturers and suppliers willing to publish electronic information. This allows fast and accurate retrieval of specified product information, as well as delivering information in a form useable by CAD systems and other design tools (e.g., thermal simulation programs).

The main parts of the system comprise the following. First, the user, who has an interface with the system directly through a web browser or indirectly through a design tool. Second, the main system or Building Object Warehouse (BOW) server. Third, the distributed product databases at numerous sites across the Internet containing product data in a variety of formats. Finally, there is provision for interaction with existing KBS systems for product advice, design considerations, etc. Again, these can be distributed across the Internet. The main points are described briefly below and the system illustrated in Figure 1.

User Environment The user can interact with the BOW system in one of two ways: either, directly through a web browser or alternatively, through a design tool. In the former case, the user interacts directly with the query handler. In the latter case the user may point to a wall and specify "window" with the design tool supplying the size parameters directly. An application built using the ARROW API residing on the user's machine accepts parameters from the design tool and then interacts with the Query Handler on the user's behalf. This is described in more detail later in this paper.

Query Handler The function of this is to help the user formulate exactly what he or she wants in terms of parameters used in the data model. The query handler contains knowledge of the product data model and so can prompt for parameters appropriate for a particular product type. There is provision for unspecified parameters, parameters within a range and exact values only, return sort order, etc. In addition, there is provision for free-text searches of the manufacturer's general product information, which is directly tied in to one query process.

Indexes, Indexers and the Search Engine The indexer has an authoritative list of all product repository addresses. Periodically these are searched, all product parameters retrieved and indexed. There are two indexes, one a structured database 
(an OO database) containing the minimum product information specified in the product model, the second for free text containing additional manufacturer information within html pages on a web server. The free text index is kept up to date using the Harvest indexer (Hardy et al, 1996).

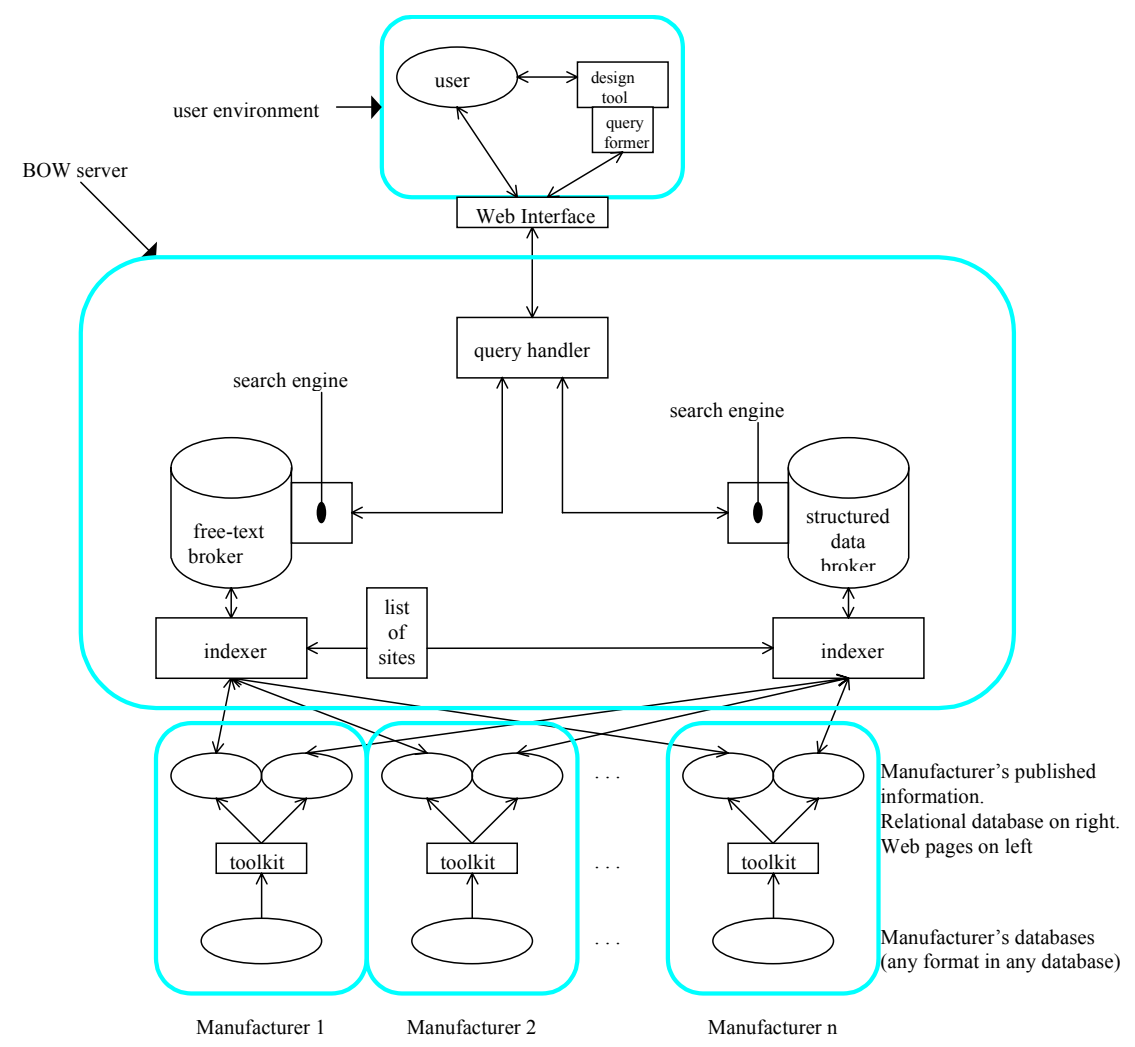

Figure 1. BOW Architecture

The results of a search when displayed on a browser consists of core information for the product together with buttons for further information, allowing the user to navigate around the full data structure. Within the data structure there is provision for electronic documents for which a mime type is stored along with the URL of the original electronic document residing on the manufacturer's machine. CAD files, VR displays etc can then easily be downloaded through the browser.

A demonstrator of the browser interface to the ARROW system is accessible at: http://www.bre.co.uk/arrow. 


\section{PRODUCT REPRESENTATION USING THE ARROW DATA MODEL}

Analysis of the requirements of the ARROW system, led to a specification of the areas that needed model development. Subsequent analysis of the existing models showed a very low level of coverage of these areas in most developments, the exception being the IAI IFCs, which gave the building blocks for almost all areas required. The final ARROW data model is therefore built entirely upon the IFCs, but with extensions to its structure to represent more specific areas. The main areas covered within the data model are:

- Product and sub-assembly structures allow for the definition of all technical data about products. The IFCs give very basic information about building elements (e.g., door, window, distribution elements), which were specialised to contain all the parameters required for product selection. This part of the specification also defines the forms of graphical representation of products and the possible connections between related products, e.g., to ducts or walls, and through which mechanism. The product section also provides the links to all associated documentation about products, in terms of BBA certificates, manufacturer's specification sheets, CAD files of product detailing, VRML file, etc.

- Organisation structures allow for the specification of manufacturers and suppliers and the connections that exist between them for specific products. There is a triangular structure which joins a product to a manufacturer, and the manufacturer to a set of suppliers, and the supplier to a set of products (and vice-versa). See Figure 2. The organisation structures provide the means for contacting manufacturers and suppliers as well as all information about their range of products and services.

- Catalogue information ties closely to products, manufacturers, and suppliers providing a mechanism to duplicate the type of catalogue services currently offered in the industry.

- Core information structures provide the majority of the basic information required in all other parts of the ARROW system. This includes: mechanisms to allow for multiple classifications for a single product; definition of materials and the layering of materials; specification of the cost for a product, and cost models which allow special relationships between suppliers and customers to be recognised; specification of standard units, and conversion factors for nonstandard units; specification of measured values (e.g., luminosity, mass, volume); and documentation types to define whether a file is a CAD detail, VRML, or a Word document and also whether it is a contract, detailed drawing, etc. This was the area which was best covered by the IFCs and required the fewest additions (the main exception being the document model specification).

No matter how complex the data model, finding appropriate fields to search upon is still a problem. To address this problem ARROW allows free-text searching of manufacturers' web pages as an integral part of the searching process. For each product, the system contains information in two forms: a) structured database product information within the above described data model, and b) a free-text 
description of the product in an associated web page. In addition to allowing more flexible querying, it also allows the manufacturer to:

- present a sales pitch for the product,

- differentiate the product from rival products, and

- describe aspects of the product not adequately covered by the formal data model.

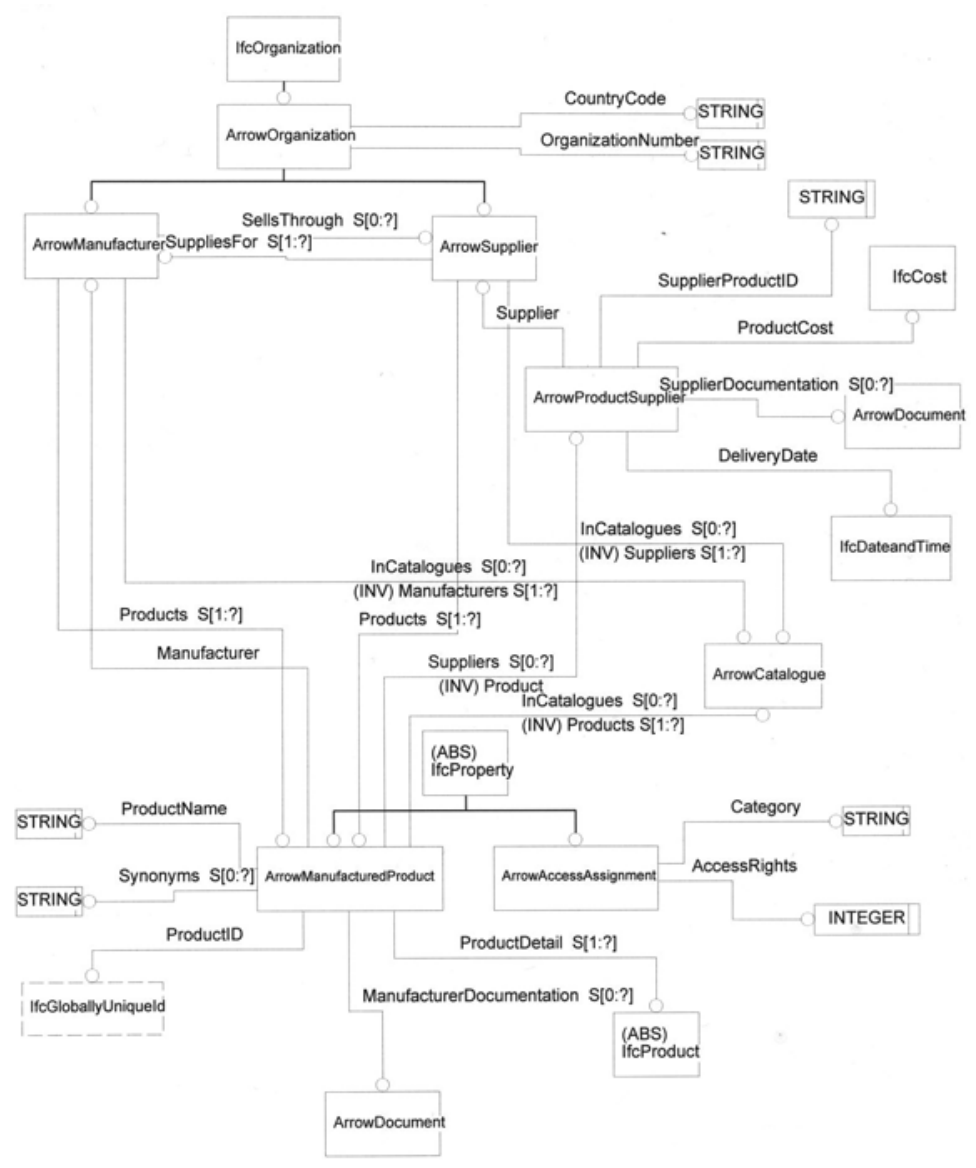

Figure 2. EXPRESS-G diagram of a portion of the ARROW Data Model

The full specification of the ARROW data model can be found in Amor et al. (1999). This document describes all structures in the data model as well as routes to implementation of the data model. Mappings to common implementation forms are also provided, e.g., SQL, C++ and Java. 


\section{ISSUES FACED IN PROVIDING INFORMATION BASED UPON STANDARDS}

The development of the ARROW system, which is concurrent with the development of international standards for the construction industry, has raised many interesting issues. The current approach to the development of data models, which are focused on specific business processes and servicing specific processes in the industry, does not provide the broad definition of information on the whole range of manufactured products which are needed to construct a building. Though there is a libraries group in the IAI, there has been little visible progress in this area over the three year timeframe of this project, which vindicates a decision not to wait for international standards to provide the required model for the ARROW system.

The ARROW system is based upon the most recent published standards (IFC version 1.5.1), however, the data model extensions required to implement the ARROW system puts ARROW in the position of creating non-standard information in its dealings with manufacturers, suppliers and user applications. Though ARROW participants can provide input to likely standards in this area there is no guarantee, and indeed a high likelihood, that the final standards are different from those in the current system. Though, as there is a limited number of measured attributes for most manufactured products, the different should not be large. The hope is that through having a semantically clear data model it will be possible to map the ARROW data structures through to those which are finally produced as standards. The current IAI standards provide a mechanism called property sets to allow user specified attributes to be attached to any object in a model. Though ARROW can provide all its information in a standards compliant file through this mechanism we have resisted following this route for all of our extensions. The main reasoning is that this seems to negate the benefit of standards, as then the consensus on semantics of property set attributes needs to be agreed with every new participant using (some subset of) the user defined attributes (this impacts all information providers and all user applications). It is also not clear how homonym conflicts between different information providers can be caught and resolved. The authors look to evolving standards in the XML world, especially RDF (Resource Description Framework) (URL1) to provide possible solutions to this problem.

In attempting to gain structured information from manufacturers and suppliers the problems with the complexity of current standards caused many problems. In short, the overhead in a manufacturer or supplier understanding enough of the current standards in order to provide their information in a form suitable for ARROW is daunting and not currently justifiable financially. Hence, the development of a simplified model for the information provides alongside toolkits to map through to the full data model is seen as the only way forward in the short to medium term for ARROW-like systems. The disparity between relational database structures, which is the most that can be expected from manufacturers and suppliers, and the object-oriented data structures of current standards also poses problems. Though it is possible to map between the two technologies this is a complex task for the majority of IT service groups in an organisation to undertake, and implementing an object-oriented model in a relational system provides an inefficient system (e.g., handling polymorphic object references). 
A similar problem of model complexity is seen at the user end. Teaching all users the meaning of all the attributes for objects in which they may wish to search is also a daunting task. However, if the semantic specification of attributes are continued to be made available electronically, along with the standards, this provides a means to take users to a clear definition of what they are dealing with. It is also likely that interfaces specialised for specific types of users will be developed (e.g., architects, HVAC engineers, etc.) providing a view which matches their understanding. This is similar to the approach already taken in providing the data from the central database to user applications. Views of the data are created which can represent, for example, IFC 1.5.1 data structures as well as the full data model.

\section{ARROW API}

The ARROW API consists of a collection of Java classes that duplicate the functionality available through the browser interface. These allow a developer to extend the functionality of a design tool so it can interact directly with the ARROW system. The functionalities of the API are:

- To return a simple YES/NO answer on whether a product with the specified parameters exists or not.

- To return a list of the products available in the database. This would usually be the first information a design tool would want.

- To return a list of the searchable fields associated with a given product. The application developer will need to know the fieldnames for a product. These include; provision for either precise or range values for numeric parameters; free-text contained within manufacturers web pages associated with products and also results sorting requirements. He can then decide whether to bind certain design parameters stored within the drawing to specific fields or whether to present some or all of the fields to the user to narrow the search.

- To return the table of results that the given query generates. The search will return details of all objects that satisfy a query. It is then up to the application developer to choose how to display these results or what to do with them. The results are returned in chunks, each being broadly associated with a class in the underlying IFC datastructure, allowing navigation through this datastructure.

- To download the specified file from the BOW Server and save it to a specified local location.

- To download the specified file from the BOW Server and returns its contents.

These last two are required for inserting any drawing file that may be stored in the database for a particular product, into a user's drawing. 
5. AUTOCAD DEMONSTRATOR

A demonstrator has been built to identify the closest matching manufactured product to a designed product and then show the direct importation of a DWG of this product into an AutoCAD drawing. The demonstrator consists of several parts:

1. AutoCAD with BS Link (URL2) installed to allow the storage of object information within the drawing.

2. An AutoLisp script.

3. The ARROW API as java classes.

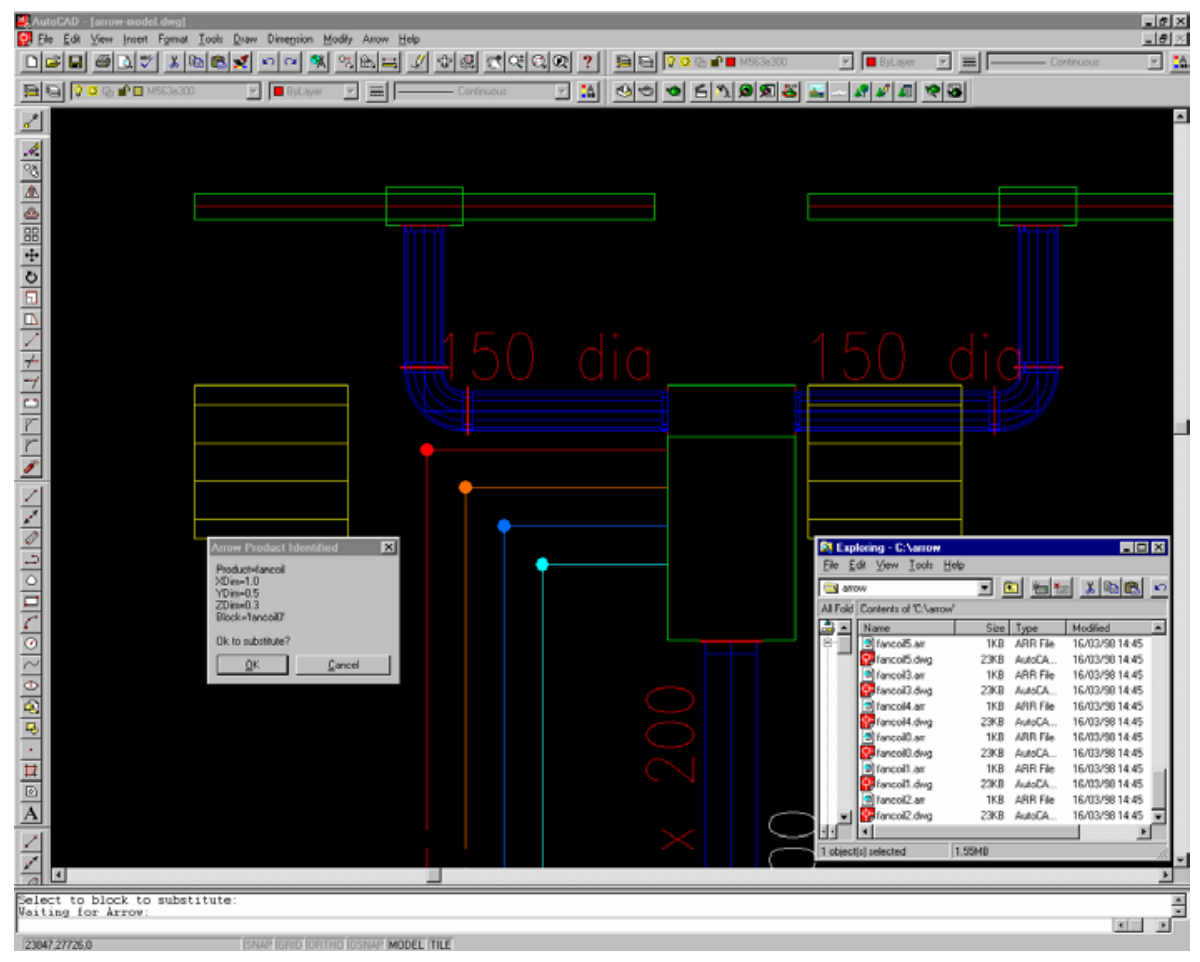

Figure 3: ARROW AutoCAD demonstrator. The user is presented with the closest match to the required fancoil unit dimensions and is being asked if he wishes to substitute the original with the manufacturer's product drawing.

The AutoLisp script provides the user interface to the ARROW system. It also reads the selected product type and dimensions from the drawing, changes the actual dimensions into a user defined acceptable range. It assembles this information into an ARROW query and passes it to the ARROW API. When the search results are returned the script selects the product with dimensions closest to those specified in the drawing. (Here the script simply looks for the product with the smallest absolute difference between sum of product dimensions and the sum of the required dimensions.) The script then submits a second query requesting the download of the DWG file for this product (if it exists) stored on the manufacturer's machine. Once 
downloaded the component drawing is automatically substituted into the main drawing.

\section{LINKS WITH UK MANUFACTURED PRODUCT RELATED SYSTEMS}

The development of the ARROW system, at the same time as other developments in manufactured product related systems, has highlighted the great overlap between the requirements of these systems. The UK National Standard Details Library (NSDL) project (URL3) has developed an infrastructure to allow access to standard details for common industry products which are usable in a number of CAD systems (e.g., DWF format to allow drag-and-drop to AutoCAD, see Figure 4). This is a process which precedes the initial selection of a manufactured product, but the attributes specified for the standard detail are the same as those then needed to select a compliant manufactured product. The same is true for the certification process. Often a manufactured product is searched for with a requirement that it has been certified as fit for use. The ARROW system already captures the link to certificates where they exist for a manufactured product, however, the certification process measures many of the attributes that a user would wish to select a product on (e.g., U-value, or fire resistance).

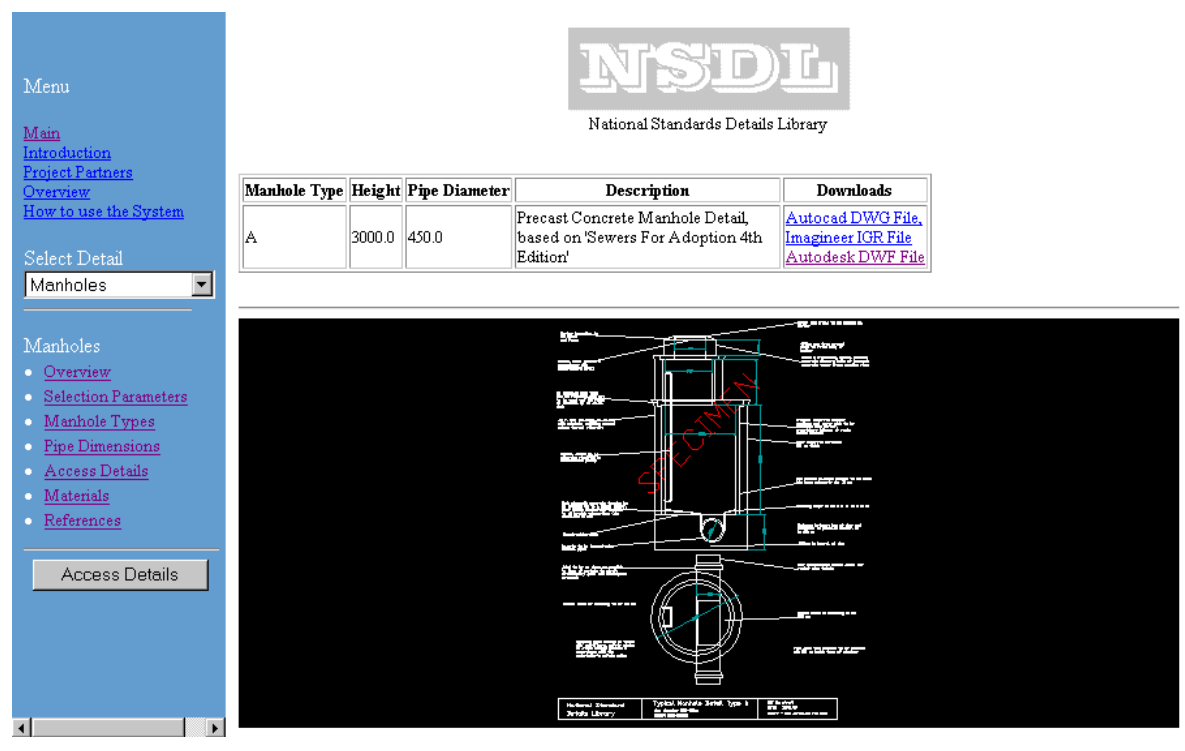

Figure 4. A DWF view of a standard detail manhole

To show the benefit of linking to related systems ARROW and the NSDL will be extended to allow the parameters used to select a standard detail to be re-used 
when finding a manufactured product. ARROW will also be extended to enable measured attributes from the certification process to be captured alongside a manufactured product description, as well as the attributes supplied by the manufacturer. The approach to these extensions is to harmonise the data representation utilised in all three systems so that attribute data can be re-used across applications.

\section{THE WAY FORWARD}

The ARROW system presented provides a view of what the future may hold. This is not an isolated view, as similar systems are being developed at both a research and commercial level in many countries. In the UK the Barbour Index (Barbour 1997) provides some measured attributes for its product catalogue as a step up from purely classification based searching for manufactured products. The model of the future that we hold is one where multiple ARROW-like systems exist in all countries in a similar way to the existence of current product catalogues, mostly aimed at different, but overlapping, segments of the market. The major difference from today's systems is that the new systems promoters will see benefit in establishing links with other systems, especially in other countries. The selling point will be access to a much greater range of manufactured products, which reflects the growing globalisation of the manufacturing market. To enable this to happen there must be broad agreement on the data models used to represent manufactured products, and there must also be agreement on a set of interface functions (API) which will make interoperability between different ARROW-like systems a possibility. In the short term these agreed models are likely to be defined ahead of the standardisation efforts. But, with an effort to build upon current and developing structures, the difference between current agreements and final standards should be small.

The largest challenge facing those developing ARROW-like systems is gaining structured data from all manufacturers and suppliers. This requires a large change in approach for the majority of manufacturers and suppliers. However, while the longterm benefits of current developments are clear, the short-term benefits are harder to identify and quantify. There will need to be a large effort expended to ensure that enough information is provided in the new form to make it a requirement for all manufacturers to publish in the new style to stay competitive. The development of toolkits for manufacturers and suppliers, to ease the cost of this process, will be a major facilitator in the development of ARROW-like systems.

The other development which will benefit the creation of manufactured product systems is the appearance of commercial design tools which utilise the standard data models now under development. These tools are starting to appear (e.g., Autodesk's Architectural Desktop with IFC interface) and with this shift to object-based systems it will be easier to interface these design tool's requirements to the information which can be offered from ARROW-like systems. 


\section{ACKNOWLEDGEMENTS}

The authors acknowledge the support of the UK government in the development of the ARROW system through their Partners in Technology programme. The manufacturers who have provided their product information are gratefully acknowledged, as well as Advanced Visual Technology who provided the industry view in the project. The ARROW system is being further developed through EU funding in the CONNET project, in co-operation with BII and VTT in Finland.

\section{REFERENCES}

Amor, R., Newnham, L., Parand, F. and Nisbet, N., 1999, The ARROW Data Model Specification, BRE publication, Watford, UK, March 99.

Barbour Index plc, The Barbour Report 1997, Electronic Delivery of Product Information, 1997.

Hardy, D.R., Schwartz, M.F. and Wessels, D., 1996, Harvest User's Manual, Technical Report CU-CS-743-94, Department of Computer Science, University of Colorado, USA.

ISO (1994) ISO/DIS 10303-1:1994. Industrial Automation Systems and Integration - Product Data Representation and Exchange Part 1: Overview and Fundamental Principles.

Newnham, L., Parand, F., Amor, R. and Nisbet, N., 1997, The ARROW Framework for Object Warehouses, CIB W78 Workshop on Information Technology Support for Construction Process Re-Engineering, IT-CPR-97, Cairns, Australia, 9-11 July, pp. 319328.

Newnham, L., Amor, R. and Parand, F., 1998, Gaining Quality Manufactured Product Information through ARROW, PDT Days, BRE, UK, 25-26 March, pp. 39-46.

Newnham, L. and Amor, R., 1998, Translation of Manufacturer's Product Data for the ARROW Product Search System, The Second European Conference on Product and Process Modelling, London, UK, 19-21 October, pp. 405-412.

URL1, http://www.w3.org/RDF/, The W3C page on the Resource Description Framework.

URL2, http://bs-link.visual-tech.co.uk/ Visual Technology's BS Link page.

URL3, http://cig.bre.co.uk/nsdl/docs/nsdl.htm The National Standards detail Library Home Page. 